Journal of Applied Mathematics and Bioinformatics, Vol. 11, No. 1, 2021, 25-31 ISSN: 1792-6602(print), 1792-6939(online)

https://doi.org/10.47260/jamb/1112

Scientific Press International Limited

\title{
Entropy-Based Evaluation of DNS Activity for Threat Hunting
}

\author{
Argyrios (Argi) Alexopoulos ${ }^{1}$
}

\begin{abstract}
The paper documents, based mainly on [1]-[5] published papers where a consistent mathematical description of cyberspace and various types of Cyber-Attacks and protection measures are presented, a mathematical approach for Cyber Threat Hunting $^{2}$ using Domain Name System (DNS) ${ }^{3}$ observations. After referring [5] to the various Advanced Persistent Threat (APT) ${ }^{4}$ hunting techniques we propose a high level, mainly, entropy-based technique for detecting the existence of various threat vectors in our networks, demystifying DNS Anomalies.
\end{abstract}

Keywords: Domain Name System (DNS), Advanced Persistent Threat (APT) actors, Entropy, Anomaly Detection.

${ }^{1}$ Cyberspace Analyst staff in International Organization, Belgium.

${ }^{2}$ https://en.wikipedia.org/wiki/Cyber_threat_hunting

3 https://en.wikipedia.org/wiki/Domain_Name_System

${ }^{4}$ https://en.wikipedia.org/wiki/Advanced_persistent_threat

Article Info: Received: April 20, 2021. Revised: May 3, 2021.

Published online: May 10, 2021. 


\section{Introduction}

The aim of the present paper is to propose an efficient threat hunting process by reducing DNS ambiguity in our internal network processes.

Based mainly on [1]-[5] published papers where a consistent mathematical description of cyberspace and various types of Cyber-Attacks and protection measures are presented, a mathematical approach for Cyber Threat Hunting using DNS observations. After referring to the various APT hunting techniques [5] we propose a high level, mainly, entropy-based technique for detecting the existence of various threat vectors in our networks, demystifying DNS Anomalies.

\section{Malicious DNS Usage}

A quite simplistic graphical display of how DNS work is shown below. We will not go to details on that since this is not the purpose of this paper and in addition, some related bits are described in the following sub chapters.

It is known and widely seen that APT's and cyber criminals utilize DNS for Command and Control $(\mathrm{C} \& \mathrm{C})$, beaconing or resolution of attacker domains ${ }^{5}$. This is the main reason why collection and analysis of DNS events are critical for hunting, detecting, investigating and responding to threats. Defenders have challenging task to figure out what are the DNS abnormalities and distinguish them for threat hunting purposes.

All DNS related interactions for C\&C communication that can be monitored and assessed in order to provide an entropy-based anomaly detection of suspicious or/and malicious activities in our network ecosystem are analysed in section 4 .
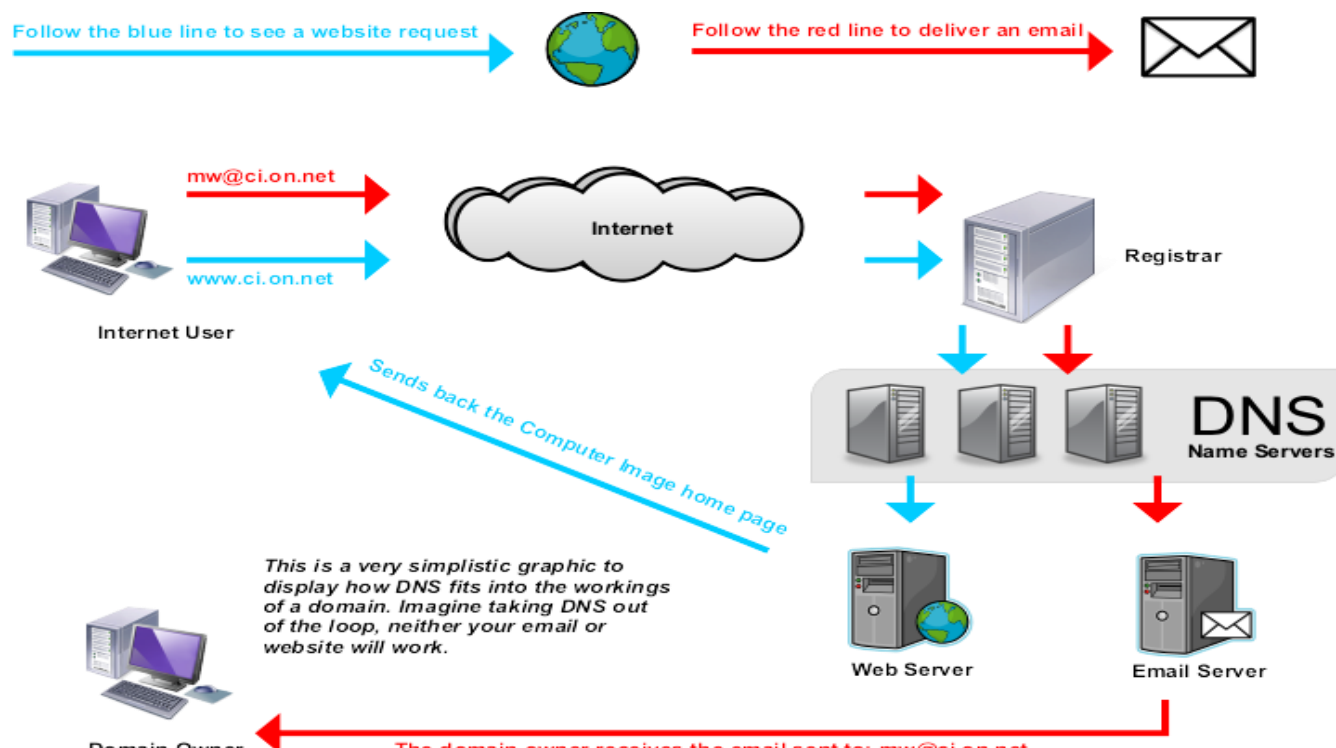

This is a very simplistic graphic to

display how DNS fits into the working

of a domain. Imagine taking DNS

website will work.

Domain Owner

The domain owner receives the email sent to: mw@ci.on.net

${ }^{5}$ https://resources.infosecinstitute.com/topic/attacks-over-dns/ 


\section{Entropy from DNS Perspective}

Entropy ${ }^{6}$ is a scientific concept, as well as a tangible physical property that is most frequently related to a state of disorder, randomness, or uncertainty. The concept itself is used in diverse fields, from classical thermodynamics, where it was first recognized, to the principles of information theory. It has found far-ranging applications in chemistry and physics, in biological systems and their relation to life, in economics, sociology, weather science, climate change, and information systems including the transmission of information in telecommunication.

In this paper, we refer to entropy from a DNS perspective and we orient it in broad DNS ecosystem. We will identify all main DNS interactions that we can measure a kind of disorder, randomness, or uncertainty and we will transfer the concept of Entropy-Based Evaluation of DNS Activity for Threat Hunting in our previous scientific research [1]-[5].

\section{DNS Interactions to be Entropy-Evaluated}

As already stated, there are many DNS interactions that malicious actors and more likely APTs will conduct to avoid detection. In this paper we focus on C\&C related traffic. Some of them will be shortly presented below. We may not be able to keep pace with every new DNS exploitation but we may be proactive by calculating a kind of entropy-based threshold. We will show holistically that all these potentially malicious activities through DNS have high Entropy value.

\subsection{DNS Queries to Unusual Destinations}

Disorder, randomness, or uncertainty increases when DNS queries to unusual destinations increases. Let $D N S_{1}$ be the queries to abnormal destinations. This abnormality may be identified internally using statistical analysis.

\subsection{DNS Traffic Bypassing DNS Forwarding Servers}

Traffic is expected to be forwarded to Internet Service Provider (ISP) or DNS Service Provider, from internal hosts, via a DNS forwarding server. Any traffic bypassing DNS forwarding Server/Entity indicates high DNS entropy. Let $D N S_{2}$ be the bypassing traffic. We should not expect the outbound DNS to be other than the IP of forwarding server.

${ }^{6}$ https://en.wikipedia.org/wiki/Entropy 


\subsection{DNS Queries Using Multiple Subdomains}

Given that DNS queries do not normally use subdomains (some exceptions exist though), DNS entropy increases proportionally to the number of dots (subdomains) of the queries. For example, the following query indicates high disorder, randomness, or uncertainty:

SV1DVV.2.8572Y8.YY.19283OLPDL20PPLPOLK.CSEC.apple.net

Let $D N S_{3}$ be the multi subdomains queries.

\subsection{DNS Queries Using Lengthy Domains}

Combination of the existence of multiple domains in DNS queries with lengthy ones is indicative of high DNS entropy and the risk of malicious activity is greater. Let $D N S_{4}$ be the lengthy DNS queries. Therefore, by combining both the character length and sub domain in a query is critical.

\subsection{DNS Queries Including a Mix of Upper/Lowercases}

Queries that include a mix of upper and lowercases indicate a higher DNS entropy, according to the approach of this paper. The more frequent interchange of upper and lowercase in a DNS query the higher potential DNS entropy. Let $D_{N}$ be the queries that mix Upper/Lowercases.

\subsection{DNS Queries Including Alphanumeric Characters}

Regular DNS queries are expected to contain only numbers, roman alphabetical letters, dots and hyphens. Not unlike the example of mixed case lettering, non-alpha numeric strings may indicate base 64 encoded strings. Which would be an unusual event. Let $\mathrm{DNS}_{6}$ be the DNS queries that include Alphanumeric characters.

\subsection{Allowed Traffic on Port 53 Inbound Transmission Control Protocol (TCP)}

This port is used for zone transfer and should only be allowed between primary and secondary DNS servers. In a perfect scenario, searching for outbound traffic over Port 53 will, at best, yield misconfigured systems. Alternatively, at worse, potentially malicious traffic. Exceptions to those IP's should be used as a trigger point for investigation. Let $D N S_{7}$ be the above DNS traffic exceptions.

\subsection{Outbound Traffic, Other than 53 and 123, Should not be User Datagram Protocol (UDP)}

Genuine services often rely on TCP. So, if any outbound communication is made to any port on the UDP, this should be a trigger point for investigation. Let $D N S_{8}$ be the above DNS exceptions. 


\subsection{Fast Flux DNS}

DNS fluxing is a technique used by attackers to hide an actual phishing or malware domain behind constantly changing compromised hosts (IP) which are acting like proxies. To accomplish this, the Time to Live (TTL) for DNS is set very low (close to $5 \mathrm{~min}$ ) so that the changes made in DNS will reflect quickly over the internet. Let $D N S_{9}$ be the DNS queries for domains, having a TTL less than 5-10 mins.

\subsection{Domain Generation Algorithm (DGA)}

Domain generation algorithms are used by many malware families to create a large number of domain names that can be used as a rendezvous point with $\mathrm{C} 2$. It can be detected based on a myriad of non-responsive DNS queries from the source towards Randomised Domains. Let $D N S_{10}$ be the non-responsive DNS queries.

\section{Entropy-Based Evaluation of DNS Activity}

Assume a node $V$ and the $\left.\left[\mathcal{C}_{\text {available }}(V)\right]\left(t_{i}\right)\right]$ that is the set of ordered columns of all available constituents $\left(\operatorname{dev}_{1}^{(V)}, \ldots, \operatorname{dev}_{m_{V}}^{(V)}, r e s_{1}^{(V)}, \ldots, r e s_{\ell_{V}}^{(V)}\right)^{T}$ of $V$, over the time $t_{i} \in[0,1]$ and $\left.[D N S(V)]\left(t_{i}\right)\right]$ that is the set of ordered columns of all DNS activity (mainly queries) of all constituents of $V$ $\left(\operatorname{DNS}\left(\operatorname{dev}_{1}^{(V)}\right), \ldots, D N S\left(\operatorname{dev}_{m_{V}}^{(V)}\right), D N S\left(\operatorname{res}_{1}^{(V)}\right), \ldots, D N S\left(\operatorname{res}_{\ell_{V}}^{(V)}\right)\right)^{T}$ over the time $t_{i} \in[0,1]$.

Let assume also the set of DNS characteristics of DNS activity ([DNS $\left.\left.(V)]\left(t_{i}\right)\right]\right)$ that have to be evaluated $\left\{D N S_{1}, D N S_{2}, D N S_{3} \ldots D N S_{10}\right\}$ as we have described in Section 4.

We will presume the following notations [2] for the sets of relative valuations of parts (fractions) of possible constituents:

$\mathcal{S}_{W} \mathbb{C}^{(\text {fraction })}(V)$

$=\left\{\left(S_{W}\left[x_{1}, x_{2}, x_{3}, t\right]\left(\operatorname{fr}\left(\operatorname{DNS}\left(\operatorname{dev}_{1}^{(V)}\right)\right)\right), \ldots, S_{W}\left[x_{1}, x_{2}, x_{3}, t\right]\left(\operatorname{fr}\left(\operatorname{DNS}\left(\operatorname{dev}_{M_{V}}^{(V)}\right),\right)\right)\right.\right.$,

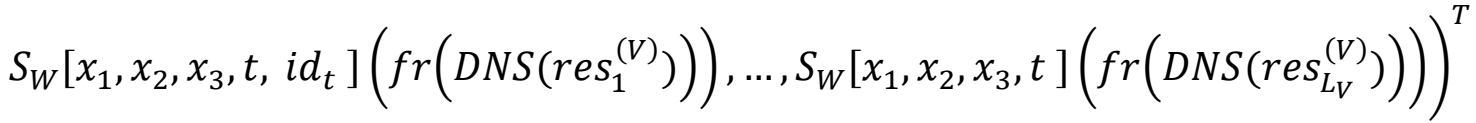

$S_{W}\left[x_{1}, x_{2}, x_{3}, t\right]\left(\operatorname{fr}\left(\operatorname{DNS}\left(\operatorname{dev}_{K}^{(V)}\right)\right)\right)$ is valuation of part

of possible DNS activity in $V$ subject to $W, k \leq \mathcal{M}_{V}$ with $\mathcal{M}_{V} \in \mathbb{N}$

$S_{W}\left[x_{1}, x_{2}, x_{3}, t\right]\left(\operatorname{fr}\left(\operatorname{DNS}\left(\operatorname{res}_{\xi}^{(V)}\right)\right)\right)$ is valuation of possible DNS activity

in $V$ subject to $W, \xi \leq \mathcal{L}_{V}$ with $\mathcal{L}_{V} \in \mathbb{N}$,

at the spatiotemporal point $\left.\left(x_{1}, x_{2}, x_{3}, t\right) \in \mathbb{R}^{3} \times[0,1]\right\}$ : 
the set of all ordered columns of relative valuations of parts (fractions) of possible DNS activity of $V$, from the viewpoint of the (user(s) of) node $W$, over the space time $\mathbb{R}^{3} \times[0,1]$;

We employed Shannon's function in order to calculate the entropy (evaluation) of $\mathcal{S}_{W} \mathbb{E}^{(\text {fraction })}(V)$ as $H(X)$ :

$$
H(X)=-\sum_{i \in X} P(i) \log _{2} P(i)
$$

where $\mathrm{X}$ is the data set $\left\{D N S_{1}, D N S_{2}, D N S_{3} \ldots D N S_{10}\right\}$ of the DNS characteristics (mainly for C\&C).

We calculate periodically for each element of $[D N S(V)]\left(t_{i}\right)$ set the $H(X)$. Afterwards, we get the mean value of $H(X)$ over the time $t_{i}$. Having in advance identify internally a "safe" threshold for DNS transactions in our ecosystem, we compare this value to the mean.

If:

- $H(X) \ll m e a n$, then the propability of a threat actor in our ecosystem is almost 0

- $H(X)<$ mean, then the propability of a threat actor in our ecosystem is relatively low

- $H(X) \approx$ mean, then the propability of a threat actor in our ecosystem is medium

- $H(X)>$ mean, then the propability of a threat actor in our ecosystem is relatively high

- $H(X) \gg$ mean, then the propability of a threat actor in our ecosystem is very high 


\section{References}

[1] Daras, N.J. (2018). On the mathematical definition of cyberspace, Theoretical Mathematics \& Applications, Vol.8, no.1, pp. 9-45. Scienpress Ltd, 2018.

[2] Daras, N.J and Alexopoulos, A. (2017). Mathematical description of cyberattacks and proactive defenses, Journal of Applied Mathematics \& Bioinformatics, Vol.7, no.1, pp. 71-142. Scienpress Ltd, 2017.

[3] Daras, N.J and Alexopoulos, A. (2017). Modeling Cyber-Security, Journal of Applied Mathematics \& Bioinformatics, Vol. 7, no.1, 2017, pp. 71-142. Scienpress Ltd, 2017.

[4] Alexopoulos, A. and Daras, N. (2018). Mathematical Study of Various Types of Cyber-Attacks and Protection, Journal of Computations \& Modelling, Vol.8, no.2, 2018 pp. 1-61. Scienpress Ltd, 2018.

[5] Alexopoulos, A. and Daras, N. (2020). Mathematical Study of Advanced Persistent Threat (APT) Hunting Techniques, Journal of Computations \& Modelling, Vol. 10, no. 2, pp. 1-24. Scientific Press Ltd, 2020. 